\title{
Effect of Ocimum Sanctum (Tulsi) Powder on Hyperlipidemic and Hyperglycemic Male Patients
}

\author{
Dr. Bhavana Chauhan \\ S.M.Patel College Of HomeScience, \\ Vallabh Vidyanagar, Gujarat, India
}

\section{ABSTRACT}

The present study was planned to investigate the effect of dry leaves powder of Ocimum Sanctum L. (Tulsi) on the Hyperglycaemic and hyper lipidemic condition. Total 40 subjects (20 hyperlipidemic male and 20 hyperglycaemic male) were selected randomly from "Manan Clinic" at Sama area in Vadodara city. Dose of 1 capsule ( $3 \mathrm{gm}$ tulsi powder) was given in early morning empty stomach and breakfast after half an hour for 45 days to each subject. The total cholesterol, triglyceride, HDL, LDL, FBS, PP2BS and HBA1C were monitored before and after supplementation of experimental capsule. After 45 days of supplementation, a significant lowering of the blood lipid profile as well as a significant lowering of the blood glucose was observed in male patients. The study suggested that Ocimum sanctum L. powder capsules had significant reduction in all report but if these capsules are given for longer period of time at least for 3months with higher doses, we get the better results.

Keywords: Ocimum Sanctum, Hyperlipidemic, CHOL, LDL, TGL, HDL, Hypoglycaemia, FBS, PP2BS, HBAIC

\section{INTRODUCTION}

Tulsi have been recommended for antidiabetic, antihypertensive adaptogenic, antilipidemic and cardio protective properties. Eugenol, the active constitute present in Ocimum Sanctum $L$, has been found to be largely responsible for the therapeutic potential of Tulsi (Devra et al., 2012). O.Sanctum contain various active constituents viz. eugenol, luteolin, ursolic acid and oeanolic acid among which eugenol content ranged from 0.175 to $0.362 \%$ (w/w). Eugeno (1-hydroxy-2-methoxy-4-allybenzene) the active constituent present in O.Sanctum has been found to be argey responsible for the therapeutic potentials of tulsi. (Sharma et al., 2013)

Hyperlipidemia is the presence of high levels of lipid in blood, including cholesterol and triglyceride in the blood. It is not a disease but a metabolic derangement that can be increase the risk of developing coronary heart disease or cardiovascular disease. Hyperlipidemia chara cterized by elevated serum total cholesterol, low density, very low density lipoprotein and decrease high density lipoprotein which are the risk factor for coronary heart diseases. Hyperlipidemia associated lipid disorders are considered to cause the atherosclerotic cardiovascular disease (Sanecha et.al., 2012).

The medicinal plants are rich in secondary metabolites, which are potential sources of drugs and essential oils of therapeutic importance. One such plant of interest is Ocimum Sanctum (Lamiaceae), traditionally used since the times of ancient civilization in India and called as the "Queen of Herbs". (Sharma S.N., 2012).

Plant based control to different diseases can serve as an alternative in the areas where there is difficulty of availability of modern treatment. In large areas of developing world, numerous plant species are used as folk medicine to Diabetes Mellitus. Many Indian medicinal plants are recommended for the treatment of Type 2 Diabetes Mellitus. 0. Sanctum, commonly known as Tulsi, is one of 
the plants that have long been used in traditional herbal medicine against Diabetes (Asolkar et al., 1992, Marles and Famsworth, 1995, Prajapati et al., 2003). However, only few scientific attempts have been made to correlate scientifically.

Hyperglycemia or high blood sugar is a condition in which an excessive amount of glucose circulates in the blood plasma. Diabetes Mellitus is the most common pathological condition that presents with hyperglycemia. Diabetes Mellitus is one of the most common metabolic disorders. It is one of the leading causes of morbidity worldwide. Diabetes mellitus is characterized by chronic hyperglycemia with disturbances of carbohydrate, fat and protein metabolism resulting from defects in insulin secretion, insulin action or both. Diabetes mellitus usually presents with its characteristic symptoms such as thirst, polyuria, blurring of vision, weight loss and polyphagia. Sometimes its acute complications like keto-acidosis or nonketotic hyperosmolarity may be the first presentation. These complications in the absence of timely treatment may lead to death. (Shaw et al., 2010)

\section{MATERIALS AND METHODS}

Study Population: The patients who visited "MANAN" clinic were included in the study. A total of 40 patients (45 to 55 years of age) were selected for the study. A Randomised control trial conducted in the "MANAN" clinic, Vadodara. The study included 20 hyperlipidemic male and 20 hyperglycaemic male patients..

Ethical Clearance: The study was performed under the supervision of the Physicians. All the Procedure was informed to the patient in his native language. After taking their consent the subjects were enrolled and interviewed at their convenient timing to fill questionnaire which is prepared for research.

Preparation and Feeding of Tulsi Leaf powder: Good quality of Tulsi leaves were purchased from local market. Wash Tulsi leaves thoroughly with distilled water. Pressed leaves between folds of tissue paper and dry at a room temperature for 35 days. The dry leaves ground to very fine powder in a mixer, Sap green colour powder were obtained. These Sap green colour powder were weighted in 3 gm each and fill in a capsule. These capsules were packed in a dry small plastic bag and seal it. Each plastic bag contains 45 capsules. The biochemical profile like total cholesterol, triglyceride, HDL, LDL, FBS, PP2bS, and $\mathrm{HbA} 1 \mathrm{c}$ were estimated at beginning and end of the intervention study. After that $\mathrm{t}$ test and $\mathrm{z}$ test were used to test the significance of mean difference between preliminary and final value of biochemical parameters.

Feeding of the Subjects: In this study tulsi powder capsule were given to the selected subjects per day in empty stomach and after 30 minute they consume their breakfast. Subjects consume this capsule for 45 days but they continued their all regular medicines that is prescribed by doctor and do not change their regular dietary pattern

Initial Assessment: The patients diagnosed as type 2 diabetes mellitus by physician of the clinic underwent physical examination. The physical examination consisted of assessment of the parameters like Height, weight, Body mass Index, Waist circumference, Hip circumference, blood pressure of extremities. Information related to pharmacological treatment (Oral hypoglycaemic agents, Insulin), pre-existing health or diabetic complications, smoking status as well as demographic information was also recorded at the initial assessment.

Laboratory investigations: Blood samples (3-5 $\mathrm{ml}$ ) were drawn from each patient by venepuncture through plastic disposable syringes. The blood samples were collected in a clean oven dried glass bottles All Laboratory investigations were conducted at central research laboratory using the set protocol and procedures established by the hospital. The total cholesterol using Glaxo India Ltd' $s$ enzymatic kit, triglycerides using Glaxo India Ltd's enzymatic kit. In the serum, low-density lipoprotein (LDL)-cholesterol and very low-density lipoprotein (VLDL)-cholesterol was precipitated by the addition of phosphor tungstic acid and magnesium chloride. The supernatant obtained was used for the determination of high-density lipoprotein (HDL)cholesterol using Glaxo India Ltd's enzymatic kit. For FBS and PP2BS they use GOD \& POD (glucose oxidase-pe roxidase) method \& for HBA1C the use enzymatic assay method. 


\section{RESULTS}

\section{Effect of Tulsi leveas (O. Sanctum) on Male hyperlipidemic experimental group}

In Male experimental group the T.CHOL decreases from $163.85 \pm 41.44$ to $148.55 \pm 43.05$ and it was insignificant at level of $\leq 0.05$ and $\leq 0.01$ (Fig-1). 5 male subjects were having noemal total cholesterol level i.e. $<200 \mathrm{mg} / \mathrm{dl}$. The TGL decreases from 165.9 \pm 137 to $140.05 \pm 114.5$ and it was insignificant at level of $\leq 0.05$ and $\leq 0.01$ (Fig-2). 12 subjects had normal triglyceride level i.e. $<150 \mathrm{mg} / \mathrm{dl}$. The LDL decreases from $97.45 \pm 40.8$ to $84.2 \pm 40.6$ and it was insignificant at level of $\leq 0.05$ and $\leq 0.01$ (Fig-3). 11 had normal LDL level $<100 \mathrm{mg} / \mathrm{dl}$. The HDL level was very slightly increases from $44.75 \pm 13.01$ to 47.4 \pm 13.02 and it was not significant at as p-value $(0.144)$ viz. $\leq 0.05$ and $\leq 0.01$. Only 4 subjects were under the normal HDL level i.e. $<40 \mathrm{mg} / \mathrm{dl}$. So we can conclude that there is no significant variation between pre-data mean and post-data mean (Fig-4)

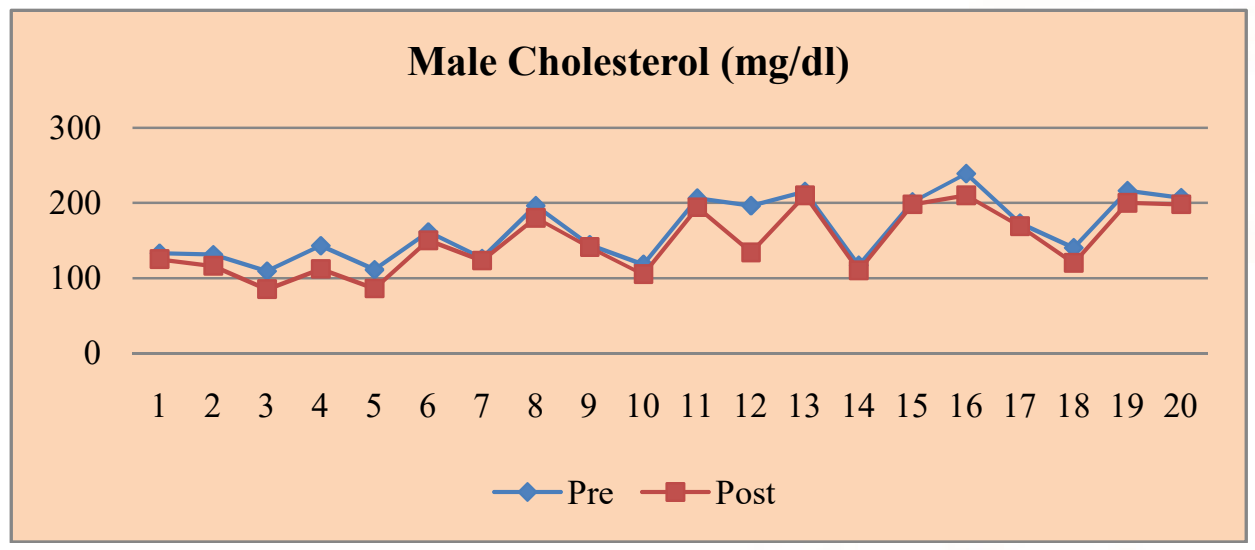

Figure 1: Comparison of Pre And Post Data of Male Cholesterol

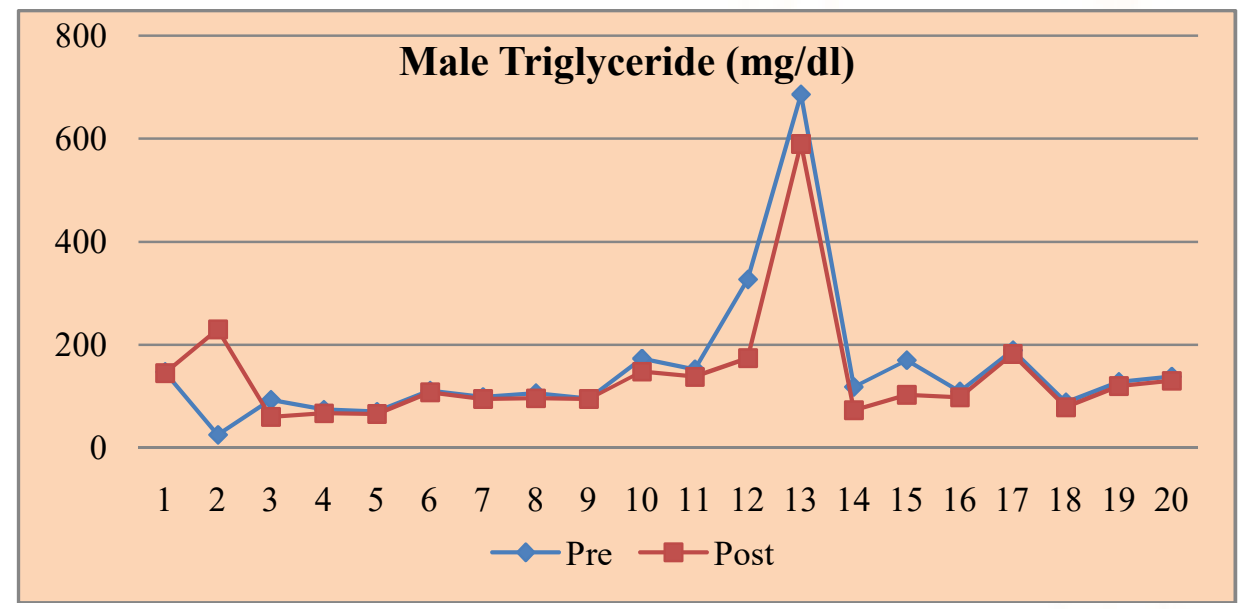

Figure 2: Comparison of Pre and Post Data of Male Tryglyceride 


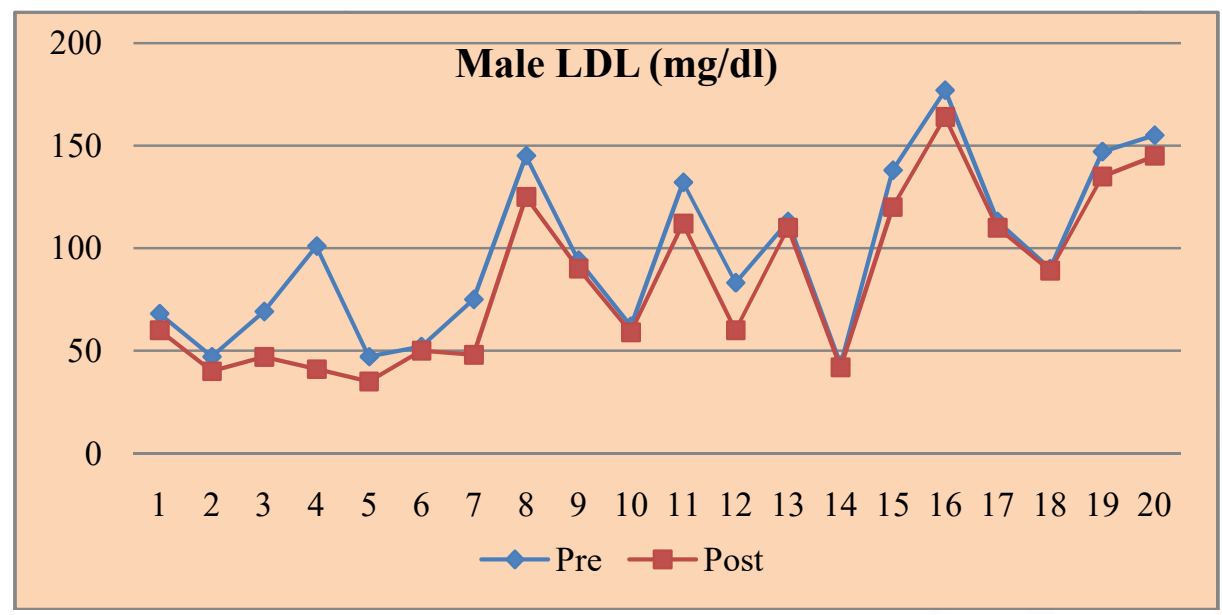

Figure 3: Comparison of Pre And Post Data Of Male LDL

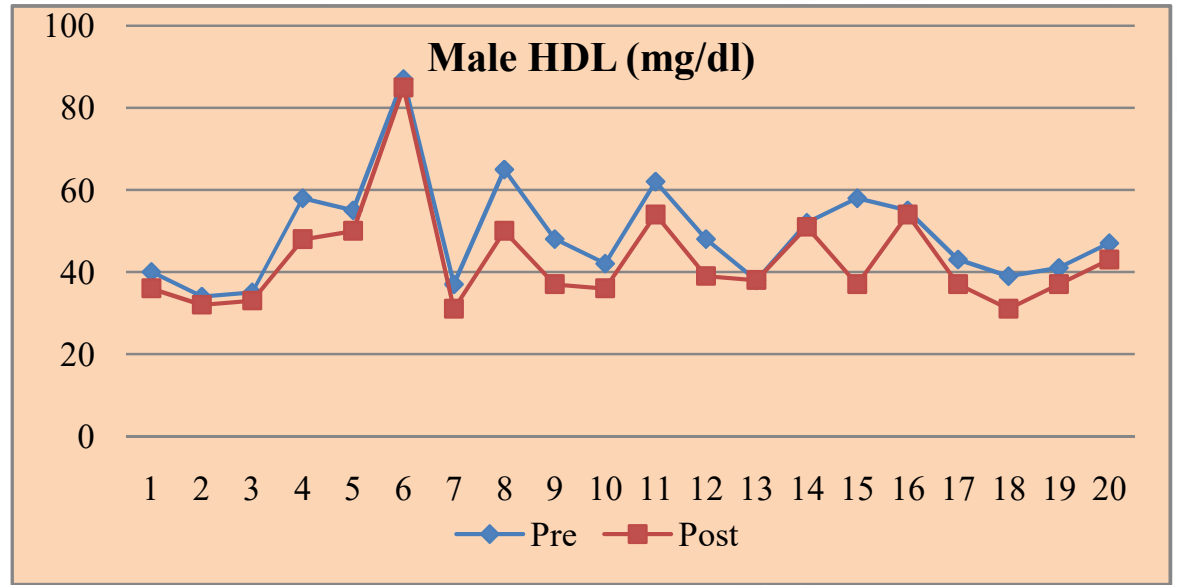

Figure 4: Comparison Of Pre And Post Data Of Male HDL

\section{Effect of Tulsi leveas (O. Sanctum) on Male hyperglycaemic experimental group}

The FBS in the male experimental group improve from the value of $388.67 \pm 13.4$ to $368.5 \pm 17.9$ and it was significant at $<0.05,<0.01$ level respectively. (Fig. 5) The PP2BS in the male experimental group improve from the value of $610.3 \pm 46.8$ to $515.9 \pm 33.3$ and it was significant at $<0.05,<0.01$ level respectively. (Fig. 6) The HBA1C in the male experimental group slightly change from $39.03 \pm 0.55$ to $38.72 \pm 0.79$ and it was insignificant. More changes were not seen in pre and post report of HBA1C due to limitation of study period of intervention. As it was only 45 days but HBA1C gives us 3 months average blood glucose level so not much change observed. (Fig. 7)

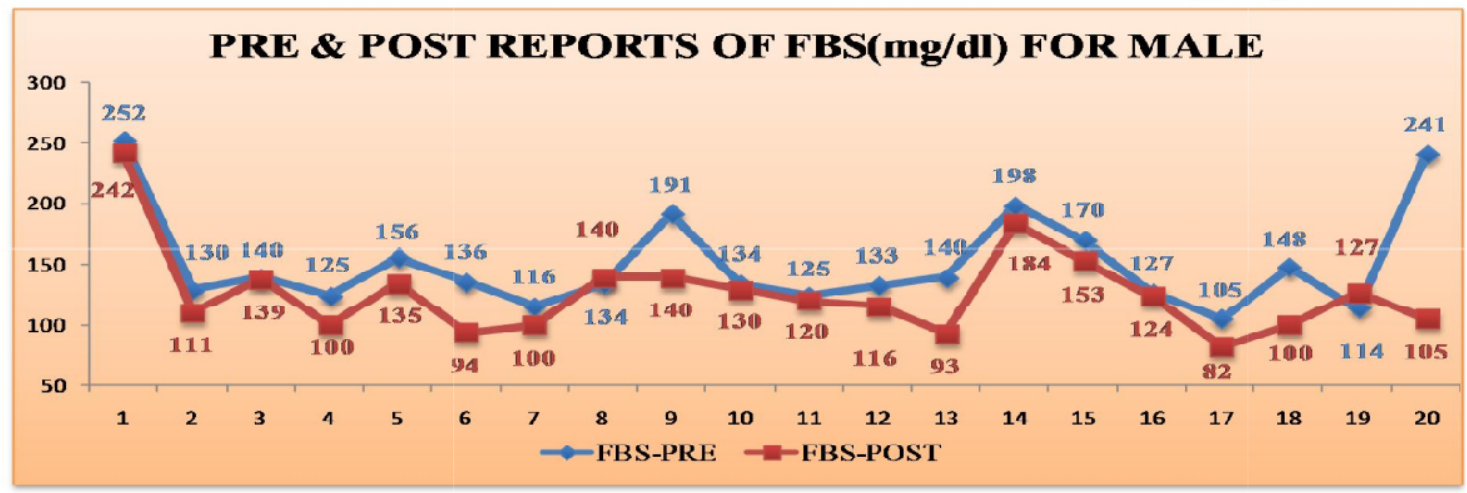

Figure 5: Comparison o Pre And Post Data Of Male FBS 


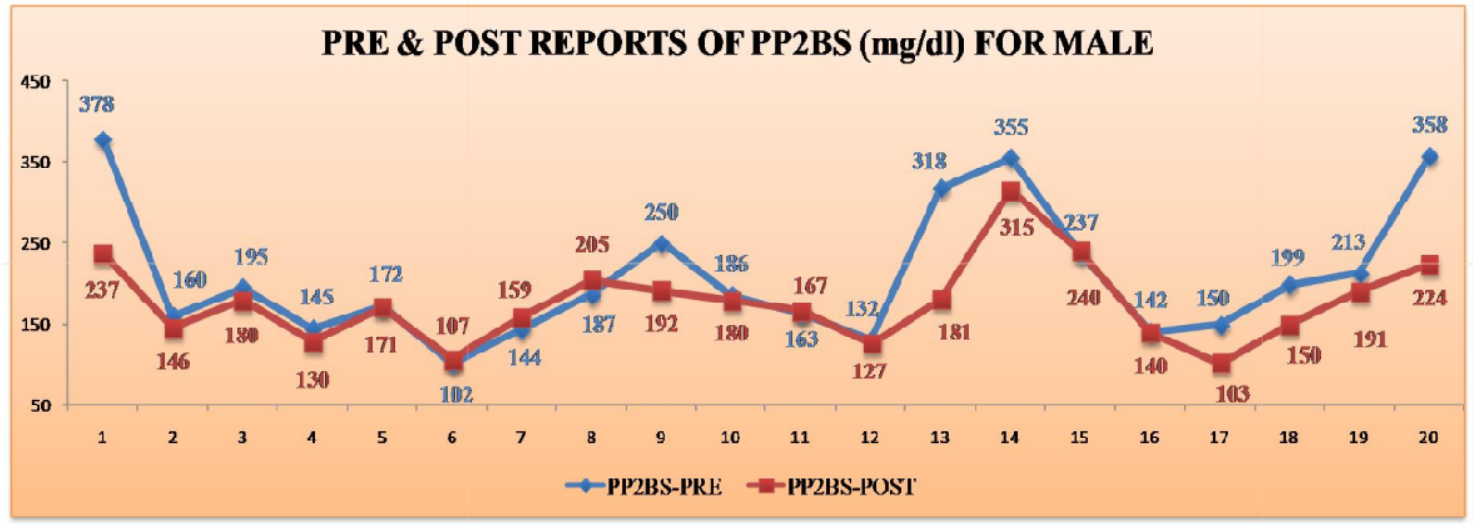

Figure 6: Comparison of Pre And Post Data of Male PP2BS

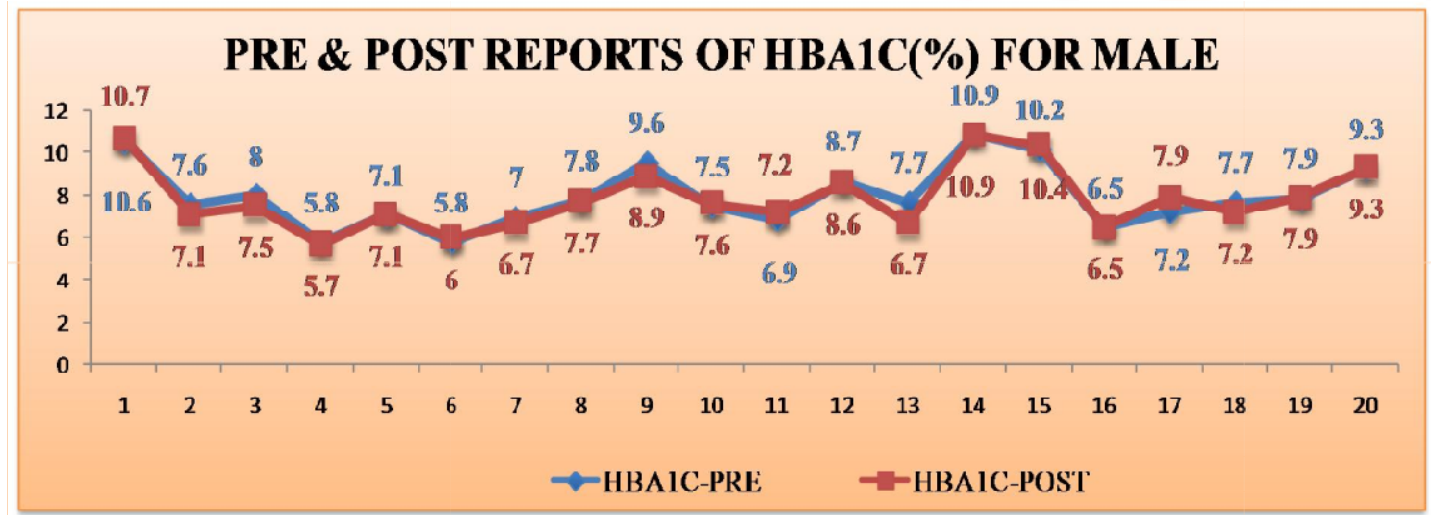

Figure 7: Comparison of Pre And Post Data Of Male HBA1C

\section{DISCUSION}

In the traditional system of Indian medicine different parts of Tulsi have been recommended for anti diabetic, antihypertensive adaptogenic, antilipidemic, cardio protective and so on properties. In this study after analysing data we conclude that Ocimum sanctum $L$. powder capsules had significant reduction in all report but giving these capsules for longer period of time (3 months), we get better results. We can also increase the dosage per day for better results. Similar study conducted by Rai et al., 1997. Tulsi powder supplementation for a period of 1 month registered a slight increase in the blood sugar and lipid levels of the controls. A significant reduction was also noticed in the levels of total cholesterol, LDL-cholesterol, VLDL-cholesterol and triglycerides after supplementation with tulasi powder. No appreciable change was noticed in the HDLcholesterol. In addition to the blood glucose and lipid levels, the total amino acids and uronic acid also exhibited a significant reduction after 30 days of tulasi supplementation. Similar study conducted by Sharma and Paul, 2011. When Ocimum Sanctum leaves were administered to DHR group, a significant hypoglycemic and antihyperlipidemic effect was observed with reduced levels of LDL, cholesterol and TGL and marginally increased HDL levels. Same result was obtained by Mondal et.al., 2012, that few individual who had an apparently normal medical history had high cholesterol and triglycerides levels. It was observed that there was a significant decrease in the elevated levels of cholesterol of these individuals.

The study conducted by Somasundaram G (2012) for 90 days after 90 days shows that significant drop in the mean FBS level from $174.35 \mathrm{mg} / \mathrm{dl}$ to $114.50 \mathrm{mg} / \mathrm{dl}$. In PP2BS after 90 days shows that significant drop in the mean PP2BS level from $247.31 \mathrm{mg} / \mathrm{dl}$ to $152.02 \mathrm{mg} / \mathrm{dl}$ and In HBA1C after 90 days shows that significant drop in the mean HBA1C level. Furthermore the results correlate with all the preclinical studies reported that who used aqueous and alcoholic extract of O. Sanctum 
in rat models showed significant decrease in the levels of blood glucose and glycosylated haemoglobin.(Narendhikaran R.T., 2006) Mitra A. (2007) suggests that tulsi leaves shows significant improvement in several biochemical parameters. Thus the composite shows hypoglycemic effect as being revealed by the reduction of fasting blood sugar level from 154 \pm 6 to $139 \pm 8 \quad(\mathrm{p}=0.020)$. Khogare et.al $(2011)$ also observed there was a significant fall in the levels of blood glucose when alloxan induced diabetic respondents was treated with tulasi level extract for 30 days and study reveals that tulasi has anti hyperglycemic action.

Suanarunsawat T. (2014) study conclude that treatment of AQOS (aqueous extracts of Ocimum sanctum L.) for three weeks significantly lowered blood glucose in DM rats. Agrawal R. (2012) conclude that the significant fall in fasting blood sugar and $\mathrm{HbA} 1 \mathrm{C}$ may be attributed to the hypoglycemic effect of Tulsi. Other studies conducted that after intervention of Tulsi leaves blood sugar level were decreased. (Dwividendra Kumar et al., 2013, Srinivas P., 2014, and JMS Hannan, 2015).

\section{CONCLUSION}

The study concludes that Ocimum Sanctum L. (Tulsi) powder supplementation to hyperlipidemic and hyperglycaemic patients gave very effective reduction in their lipid profile and blood glucose level. If the supplementation amount and period will be increased up to 3 months we may get the better result.

\section{REFERENCES:}

1) Agrawal R.. (2012) Effect of Tulsi (Ocimum sanctum Linn) on clinical and biochemical parameters of metabolic syndrome, Journal of Natural Remedies, 12(1), 63-67

2) Asolkar L.V., Kakkar K.K., Chakre O.J. (1992) Second supplement to glossary of Indian Medicinal, Plants with active principals, Part I \& Part II, New Delhi: CSRI.

3) Devra D.K., Mathur K.C., Agarwal R.P., Indrabhadu, Goyal S. and Agarwal V. (2012), "Effect of Tulsi (Ocimum Sanctum Linn.) on clinical and biochemical parameters of metabolic syndrom", Journal of Natural Remedies, 12(1), 63-67.

4) Dwividendra Kumar Nim (2013) Clinical evaluation of anti-hyperglycemic activity of Boerhaavia diffusa and Ocimum sanctum extracts in streptozocin induced T2DM rat models, Int J Pharm Biomed Sci., 4(1), 30-34

5) Hannan J.M.A. (2015) Actions Underlying Antidiabetic Effects of Ocimum sanctum Leaf Extracts in Animal Models of Type 1 and Type 2 Diabetes, European Journal of Medicinal Plants 5(1): 1-12.

6) Khogare D.T. and Lokhande S.M. (2011) Effect of Tulsi (Ocimum Sanctum) on Diabetes mellitus, Indian Streams Research Journal Vol. 1(II), 189-191

7) Marles .J., Farnsworth N.R. (1995) Antidiabetic plants and their active constituents. Phytomedicine 2, 137-189.

8) Mondal S., Mirdha B.R., Pathi M.M. and Mahapatra S.C. (2012), "Dried leaf extract of Tulsi (Ocimum sanctum Linn) reduces cardiovascular disease risk factors: Results of a double blinded randomized controlled trial in healthy volunteers", Journal of Preventive Cardiology,1(4), 177-181.

9) Narendhikaran R.T. (2006) Biochemical evaluation of antidiabetogenic properties of some commonly used Indian plants on streptozotocin induced diabetes in experimental rats. Clinical and Experimental Pharmacology and Physiology, 33(12), 1150-1157.

10) Prajapati N.D., Purohit S.S., Sharma A.K., Kumar T.A. (2003) Handbook of Medicinal Plant- A complete source book. Agrobius (India).Jodhpur: Shyam.

11) Rai V, Iyer Uma and Mani U. (1997), "Effect of Tulasi (Ocimum Sanctum) leaf powder supplementation on blood sugar levels, serum lipids and tissue lipids in diabetic rats", Plant

Foods Hum Nutr, 50(1), 9-16.

12) Sanecha C., Sharma P. K., D'Sauza U. P. and 
Shastry C.S. (2012) "Anticholesteremic and

Antilipidemic activity of Stem bark extracts of Moringa olefera in Diet induced hyperlipidemia model in rats", International Journal of pharmaceutical and chemical sciences, 1 (3), $916-923$

13) Sharma Nidhi, Sharma Preeti, Jasuja Nakuleshwar and Joshi Sureshchand (2013), "Hypocholesterolemic and antioxidant potentials of some plants and herbs", Research and Reviews, Journal of Zoological Sciences, 1(2), 26-42.

14) Sharma S.N. (2012), "A mine of medicinal uses: Ocimum Sanctum the holy basil", International Journal of Pharmacy Review and Research, 2(2), 69-74.

15) Sharma Vikram and Paul Arindum (2011), "The effect of fresh Ocimum Sanctum Linn (Tulsi) leaves on wound healing in non-diabetic and diabetic hyperlipidemic diabetic rats", Asian Journal of Biochemical and Pharmaceutical Research, 3(1), 399-404.

16) Shaw J.E., Sicree R.A., Zimmet P.Z. (2010) Global estimates of the prevalence of diabetes for 2010 and 2030. Diabetes Res Clin Pract 87, 4

17) Somasundaram G. (2012) Evaluation of the Antidiabetic effect of Ocimum sanctum in type 2 Diabetes Patients, International journal of Life science \& Pharma Research, 2(3)

18) Srinivasa Prasadacharyulu (2014) Leaves of Ocimum Sanctum [LOS]: A Potent Antidiabetic Herbal Medicine. ijird, Vol 3(4)

19) Suanarunsawat T. (2014) Anti-Oxidative, AntiHyperglycemic and Lipid-Lowering Effects of Aqueous Extracts of Ocimum sanctum L Leaves in Diabetic Rats, Food and Nutrition Sciences, 5, 801-811 